\title{
Transition from quasiperiodicity to chaos just before sawtooth crash in the ASDEX Upgrade tokamak
}

\author{
V. Igochine ${ }^{1}$, O. Dumbrajs ${ }^{2,3}$, H. Zohm ${ }^{1}$ and ASDEX Upgrade Team ${ }^{1}$ \\ ${ }^{1}$ MPI für Plasmaphysik, Euratom-Association, D-85748 Garching, Germany \\ ${ }^{2}$ Helsinki University of Technology, Association Euratom-Tekes, P.O.Box 2200, FIN-02015 HUT, Finland \\ ${ }^{3}$ Institute of Solid State Physics, Association Euratom-University of Latvia, Kengaraga Street 8, LV-1063, Riga, Latvia
}

\begin{abstract}
In this paper we investigate the sawtooth crash in the ASDEX Upgrade tokamak and present evidences supporting the hypothesis of stochastization of magnetic field lines during the crash. We demonstrate on the basis of the soft X-ray and electron cyclotron emission measurements that during the pre-crash phase the quasiperiodic transition to chaos occurs. Magnetohydrodynamic oscillations with two frequencies develop before the crash. Consistent with the most energetically favorable transition from quasi-periodicity to chaos, their frequency ratio is close to the conjugate golden ratio $G=f_{2} / f_{1}=(\sqrt{5}-1) / 2 \approx 0.618$. We think that the sawtooth crash has a universal stochastic character and it would be worthwhile to search for these transition signatures in other tokamaks.
\end{abstract}

\section{Introduction}

In magnetically confined fusion plasmas, a variety of magnetohydrodynamic (MHD) instabilities can occur, driven by gradients of kinetic pressure or current density. The sawtooth oscillation is one of the fundamental instabilities in tokamaks which is often observed but still has no definitive explanation for crash process. This phenomenon is characterized by a repetitive and rapid crash of the central electron temperature [1]. The Kadomtsev model [2], in which the $(\mathrm{m}, \mathrm{n})=(1,1)$ island turns to a new magnetic axis after the reconnection process, has provided a starting point for understanding the sawtooth, but not for explanation of the phenomenon. Several experiments show that this model is in clear contradiction with experimental observations. For instance, it can explain neither the measured safety factors $[3,4,5]$, nor the existence of $(1,1)$ mode after the crash [6,7]. As a result, a number of different theories were proposed to explain the crash dynamics and the fast crash time. Recent 2D ECE measurements $[17,18]$ of the crash allow one to prove some predictions of these models. It was demonstrated that a sawtooth crash is localized in the toroidal direction which immediately withdraws all symmetrical hypotheses like Kadomtsev model or quasiinterchange model [19]. The other, ballooning theory [20], suggests a much broader region for the crash and preferable position at the low-field side of the tokamak which is in clear contradiction with the measurements which show no preferential poloidal location. Other proposed models are based on the idea of stochastization of magnetic field lines during the sawtooth crash $[8,9]$. This variant of the crash requires no preferable poloidal position for the crash and can be also non-symmetric in the toroidal direction. The important parameters for such scenario are: (i) amplitude of the perturbations; (ii) safety factor profile; (iii) number of perturbations with different helicities; (iv) coupling of the perturbations. It was shown recently that amplitudes of the primary $(1,1)$ mode together with its harmonics are sufficient to stochastize the region if the central $q$ is less than $0.85-0.9$, which is in good agreement with measurements of the safety factor profile and allows one to explain the existence of the mode after the sawtooth collapse [7]. In this work influence of first three parameters were investigated with the field line tracing technique. The last condition, coupling of perturbations, cannot be described by means of this technique because in this type of analysis all resonances are coupled by definition. In reality, different resonances have different rotation frequencies which screen perturbations from each other and strongly reduce the 
amplitude of the magnetic fluctuations in tokamaks. Such a screening effect vanishes only if the perturbations are coupled to each other. It is obvious that the primary $(1,1)$ mode is coupled to its harmonics $(2,2)$ and $(3,3)$, but the coupling to other low order rational surfaces is not trivial and requires special investigation. In contrast to our earlier work [7], here we focus our attention on the dynamics of the instability just before the sawtooth crash and investigate transition to the stochastic stage. We present a clear indication of the transition into stochastic (chaotic) stage which supports the stochastisation hypothesis of the sawtooth crash. The stochastization can also explain the fast heat transport from the plasma core during the crash [21].

\section{Identification of the transition to stochastic phase}

As was mentioned before, we think that evolution of the $(1,1)$ instability leads to stochastic stage during the sawtooth crash. The stochastic stage is very short (20-100 $\mu \mathrm{s}$ in ASDEX Upgrade) and it is accompanied by the strong reduction of the temperature due to the heat flow in the stochastic magnetic field. Thus, all temperature measurements show only decrease of the plasma temperature which is a standard signature of the sawtooth crash from Soft X-ray (SXR) and electron cyclotron emission (ECE). Due to these problems, the stochastic phase itself can not be resolved and investigated. At the same time, transition to stochastic/chaotic stage can be checked if one uses time traces from the SXR and ECE diagnostics. These two diagnostics give two independent measurements of the temperature perturbations as line integral measurements (SXR) and as local temperature measurements (ECE). We use these signals to identify the type of transition to the stochastic stage.

Intensive investigations of completely different mathematical and experimental chaotic systems demonstrate that there are three possible roads to chaos [10]: (i) period doubling, when the period is doubling many times during the transition (ii) intermittency, which is characterized by sudden changes from non-chaotic to chaotic behavior and back; (iii) quasiperiodicity which is characterized by appearing of two incommensurable frequencies [12]. Each of these roads to chaos has a set of unique signatures which appears in the system independently from its nature. It could be a physical, biological or any other system, but the roads remain the same $[16,10,13,14]$. Thus, a set of transition signatures is a universal invariant which could be used to verify the nature of the system and clarify the type of the transition to chaos. Analysis of the experimental SXR and ECE signals before a sawtooth crash shows neither period doubling (power spectrum at frequencies $f_{1} / 2^{n}, n=1,2,3, \ldots$ with decreasing amplitudes), nor sudden jumps between chaotic and non-chaotic behavior. This eliminates the roads to chaos via period doubling or intermittency. The characteristics of the quasiperiodic transition are different. "In the quasiperiodic regime, an experimental spectrum is a series of peaks at all integer combinations of two incommensurate frequencies $f_{1}$ and $f_{2}$."[12]. To understand this type of transition we assume for a moment an arbitrary system which has two basic frequencies $f_{1}$ and $f_{2}$. Such system would have a trajectory in 3D-phase space which is lying on the torus. In case of the rational ratio between the two frequencies $\left(f_{1} n=f_{2} m\right.$, with integers $n$ and $m$ ), the system has periodic behavior (it repeats itself after a fixed period). Poloidal cross-section of the torus is Poincare plot of the system which consists of a set of repetitive points on a curve. The system trajectory is closed in this case and does not cover the torus surface completely. If the two frequencies are incommensurate, the torus surface would be cover completely and the Poincare points will never (in principle) repeat. Eventually, the Poincare points fill in the curve in the Poincare plane. In such situation, an arbitrary small change in the system (third frequency, frequency locking, etc.) destroy the surface of the torus and convert the motion into 3D motions in the phase space which is chaotic motion. It was demonstrated both numerically and experimentally that the more 
irrational relation between the frequencies, the more easy such a system goes over to the chaotic stage and computation experiments often use the conjugate golden ratio $(G=(\sqrt{5}-1) / 2)$ which is the most irrational number, since it has the slowest convergence [10].

We investigate several typical H-mode discharges which were performed during 20062007 campaigns $\left(\mathrm{I}_{\mathrm{p}}=0.8 \mathrm{MA}, \mathrm{P}_{\mathrm{NBI}}=5 \mathrm{MW}\right.$, with and without $\mathrm{ECRH}$ power $)$. We analyzed the central SXR signal (2MHz sampling frequency, $500 \mathrm{kHz}$ upper filter frequency) before a sawtooth crash in ASDEX Upgrade by means of spectral analysis and reconstruction of trajectory by means of delay coordinates which are the standard techniques for stochastic systems and have been used for identification of the transition to chaos in different physical systems $[11,15]$.
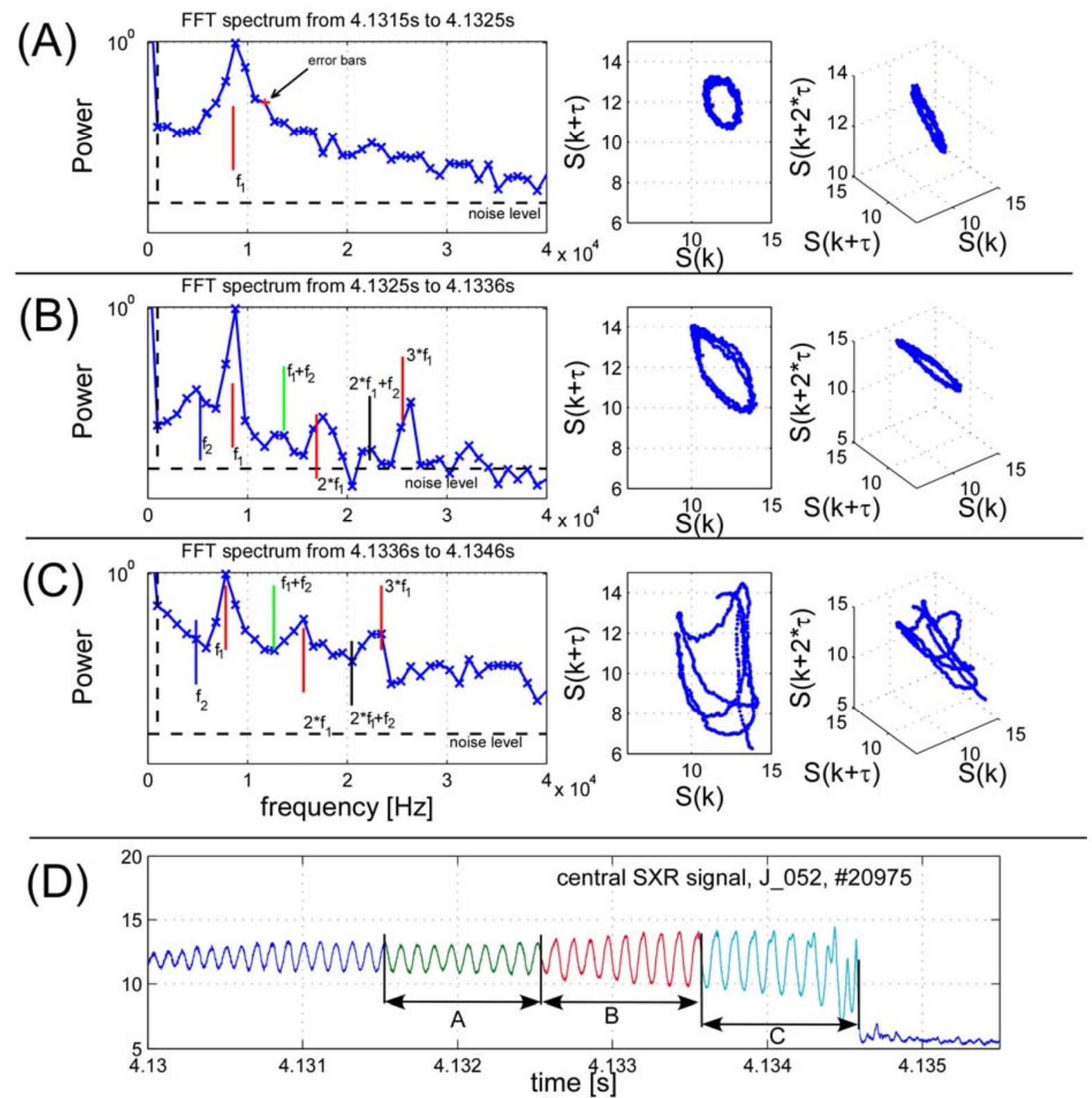

Figure 1. Analysis of the sawtooth crash in ASDEX Upgrade (A-D). Normalized spectral amplitude and reconstruction of trajectory by means of delay coordinates (number of time points for the time delay: $\tau=600)$ are shown for single mode regime $(A)$, slightly quasiperiodic regime which is close to the crash time (B), and strongly quasiperiodic regime just before the sawtooth crash (C). Smallest resolvable frequency and noise level are indicated by dashed lines. The SXR signal is shown in figure (D). 
One can clearly see a transition of the system from the single frequency state (Fig. 1A) to the slightly quasiperiodic state (Fig.1B) and then to the strongly quasiperiodic state (Fig. 1C) where the whole lower part of the spectrum is strongly enhanced and only the strongest frequencies can be seen. Such increase of broad band low frequency noise is typical, when the chaos is about to appear [10]. The frequency locking (mode coupling) occurs in the stochastic stage which is accompanied by the strong reduction of the temperature ${ }^{*}$. The frequency of the primary $(1,1)$ kink mode is marked here as $f_{1}$. (The signal itself and all stages are shown in Figure. 1D.) Another important observation is the ratios between the two primary frequencies before the sawtooth crash in Figures 1B. It is equal to the conjugate golden ratio $f_{2} / f_{1}=0.59 \pm 0.03$ (within error bars of the measurements). To show the "ideal situation" we adjust in Figure 1 position of the primary peak $f_{1}$ to the $(1,1)$ mode frequency and marked automatically all other frequencies by using the golden mean ratio and linear combinations of the frequencies. One can see that the frequency marks fit all the experimentally measured peaks within error bars of the measurements. The lowest resolvable frequency $977 \mathrm{~Hz}$ (fundamental frequency of the Fourier transform) is shown together with the noise level by dashed lines. Thus, the low frequency spectrum is completely described by two primary frequencies and their linear combinations. In typical experiments with transition to chaos, the scan of the frequencies is made to obtain the irrational frequency ratio [14,23]. In our case, the most irrational frequency ratio develops naturally in the system. This fact indicates that the chaos in the system is approached in the most "intense" way (the most robust way). Other conformation of the transition comes from reconstructions of trajectories by means of delay coordinates in 2D and 3D phase space. These plots were constructed from the same signal using the fixed time delay $\tau$ [13] and are analogous to Poincare plots (2D) and torus phase structures (3D) in phase space which were discussed before. In these phase plots our transition to chaos should have the following steps: (single frequency) $\rightarrow$ (2D torus) $\rightarrow$ (3D chaos). Indeed, in the case of the single frequency $f_{1}$ we observe a planar periodic cycle which is typical signature of pure periodic behavior (Fig.1A). In the slightly quasiperiodic case open orbits are observed (Fig. 1B). In the last pre-crash phase strongly quasiperiodic behavior is seen which is characterized by a non planar 3D structure (Fig.1C) but the torus structure is not completely destroyed. (Completely chaotic stage is characterized by a cloud of the trajectory points in 2D plot and an attractor structure or cloud of points in 3D plot depending on system's nature.) The analysis of the ECE temperature signals (32 kHz sampling rate, $16 \mathrm{kHz}$ Nyquist frequency) in the slightly quasiperiodic stage shows the same frequency spectra as in the SXR signal and it also consists of the same frequencies $f_{1}=8.7 \mathrm{kHz}$ and $f_{2}=5.26 \mathrm{kHz}$ $\left(f_{2} / f_{1}=0.605 \pm 0.025\right)$. These measurements resolve also other $\left(f_{1}-f_{2}\right)$ and $\left(2 f_{1}-f_{2}\right)$ resonances as shown in Figure 2.

\footnotetext{
${ }^{*}$ It is interesting to compare our findings with the transition to chaos in a crystal [11]. Approach to chaos in two completely different physical systems is almost identical. The only difference is that we in contrast to [11] can not resolve the stochastic stage itself.
} 

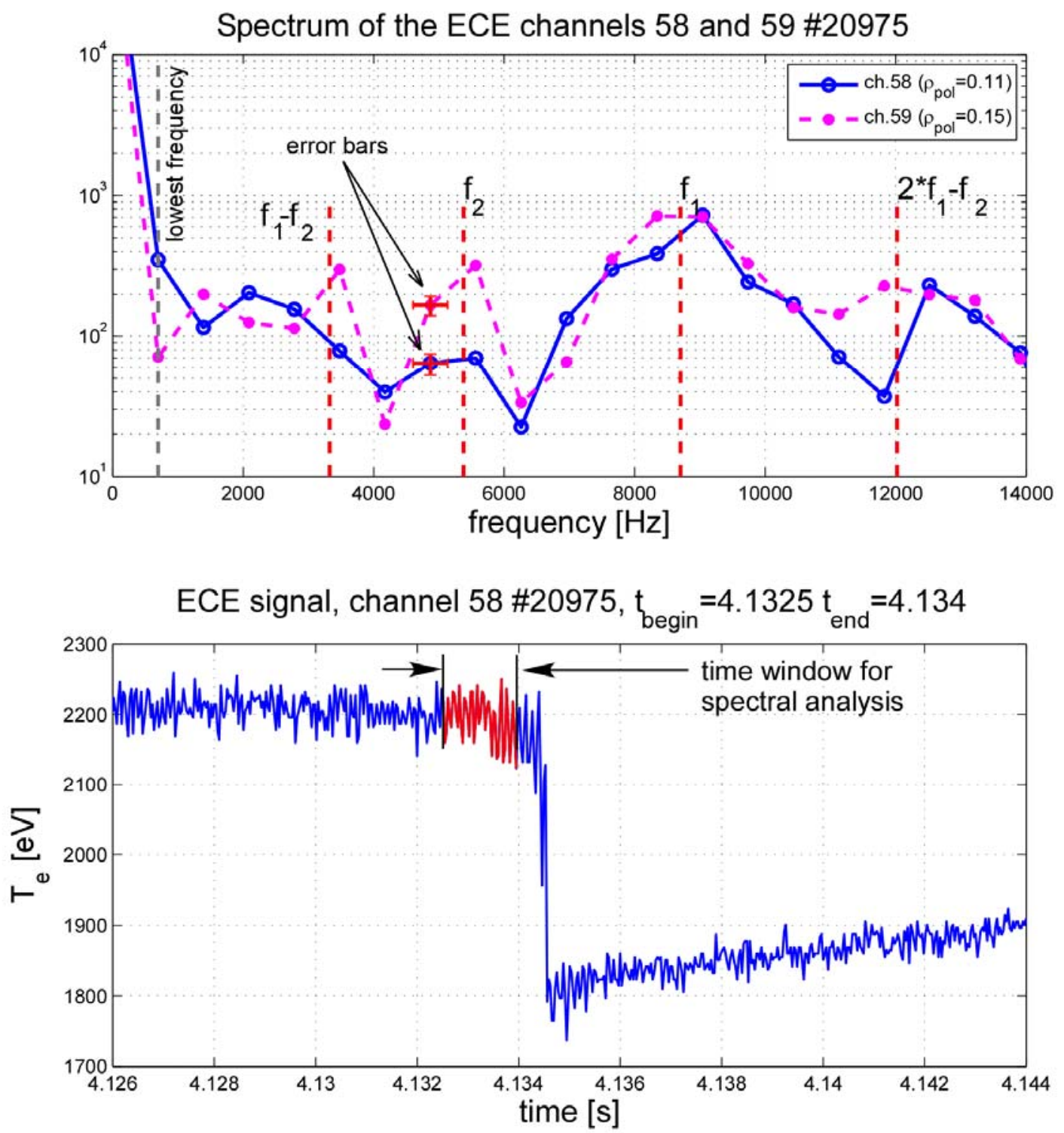

Figure 2. ECE power spectrum for two neighbouring channels and the one of the signals for the same sawtooth crash as shown in figure 1. The two frequencies are shown and the linear combination of these frequencies can be seen $\left(f_{2} / f_{1}=0.605 \pm 0.025\right)$. The lowest resolvable frequency is marked.

As an example of the universality of the frequency ratio we show the spectrum for another discharge in ASDEX Upgrade (Fig.3). 


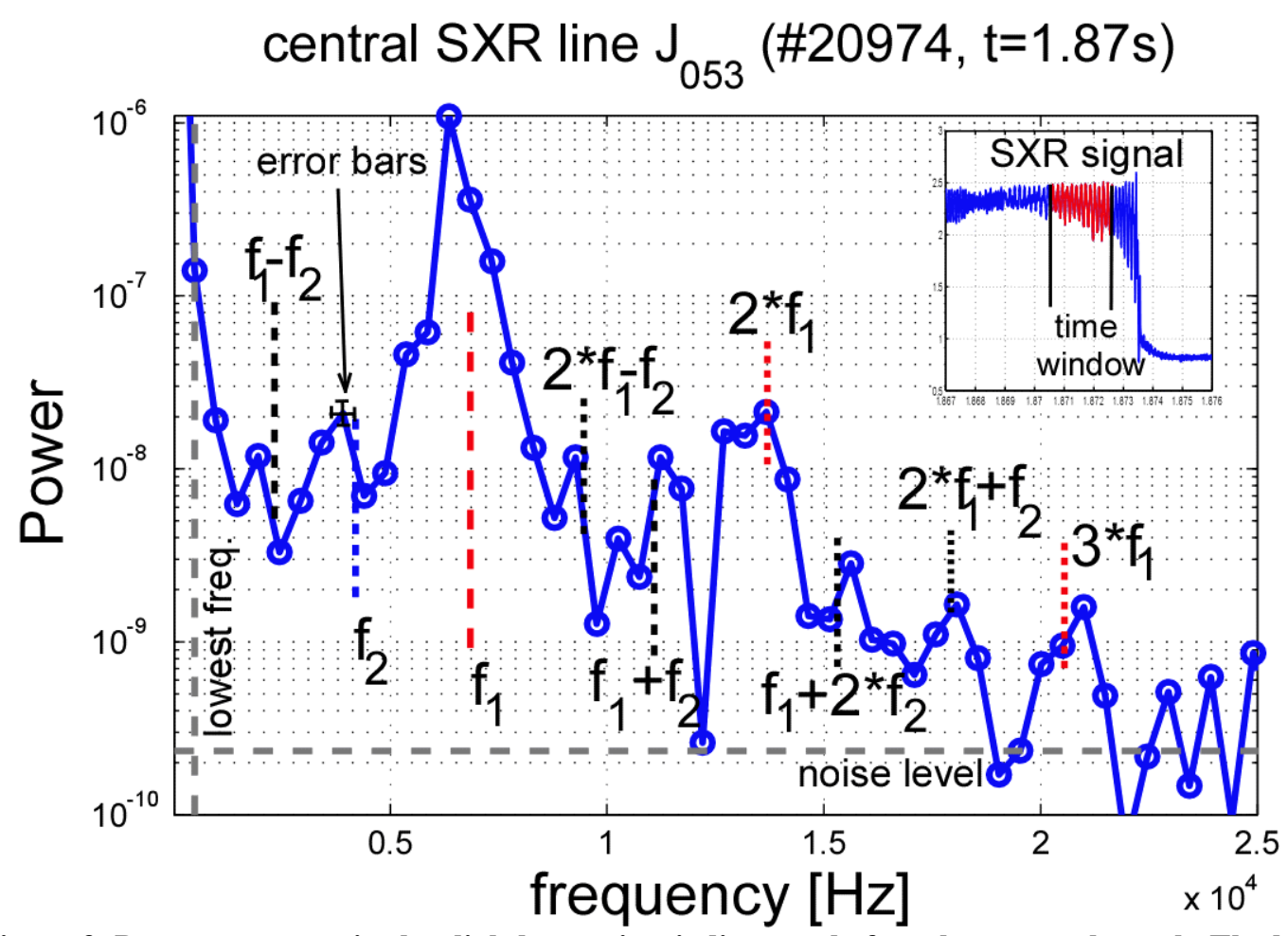

Figure 3. Power spectrum in the slightly quasiperiodic stage before the sawtooth crash. The low frequency part of the spectrum consists only of two frequencies $\left(f_{1}=6.59 \mathrm{kHz}\right.$ and $f_{2}=3.93 \mathrm{kHz}$, $\left.f_{2} / f_{1}=0.596 \pm 0.022\right)$ and their linear combinations. The ratio between the primary frequencies is equal to the golden mean. (The lowest resolvable frequency in this case is $488 \mathrm{~Hz}$.) The Soft $\mathrm{X}$-ray signal is shown in a small figure.

One can see that the frequency spectrum is described by the predicted linear combinations of the frequencies and the conjugate golden mean ratio between the frequencies (within error bars of the measurements). Depending on the duration of the slightly quasiperiodic phase, different number of the resonances can be resolved (longer time interval in Fourier analysis provides better frequency resolution). We have found that longer $(1,1)$ precursor phase before the crash typically corresponds to a longer phase of the quasiperiodic motion. All these observations strongly support the hypothesis of the quasiperiodic transition to chaos during the sawtooth crash. 
FFT amplitude, I-camera, \#20975, $\mathrm{t}=4.13 \mathrm{~s}$

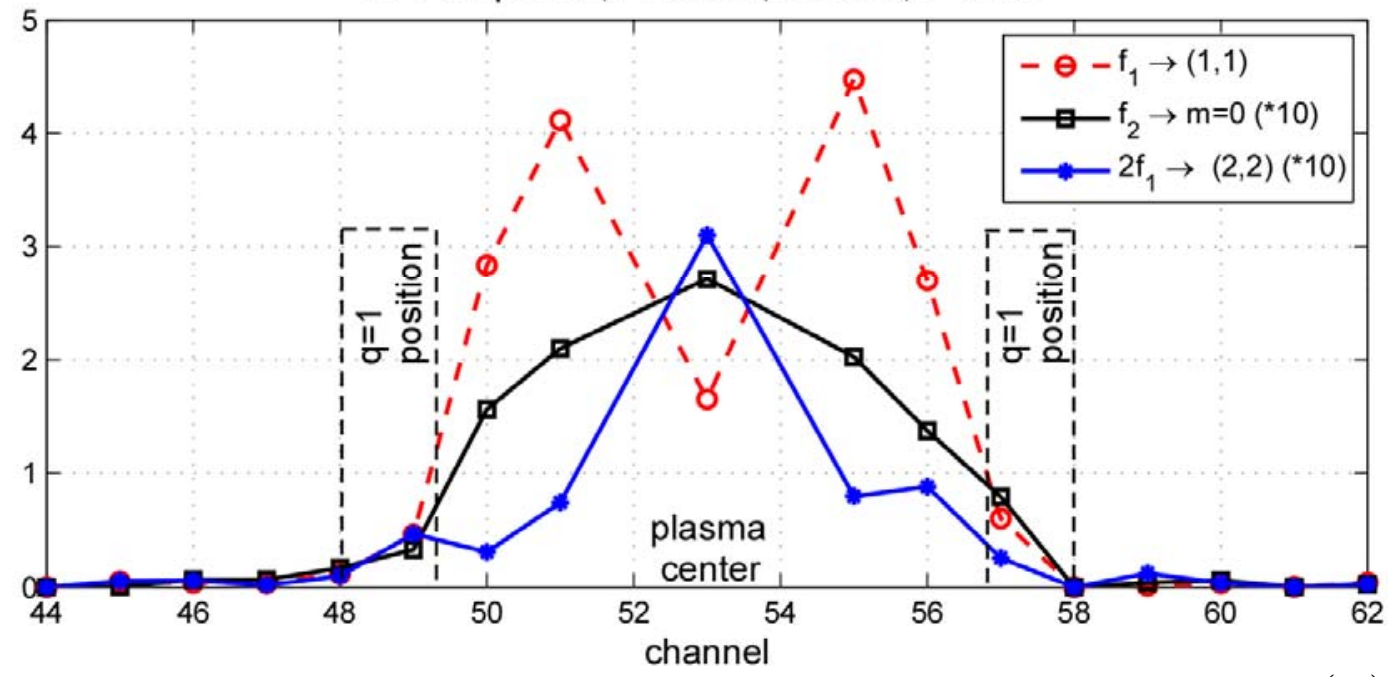

Figure 4. Experimental FFT amplitude for the same sawtooth as in Figs.1 and 2. Primary frequency $\left(f_{1}\right)$ shows the strongest signal and a clear $(1,1)$ structure. Second harmonic $\left(2 \cdot f_{1}\right)$ has $(2,2)$ structure (amplitude is multiplied by factor of 10 in the figure). Second frequency $\left(f_{2}\right)$ shows $m=0$ structure (amplitude is multiplied by factor of 10 in the figure). Position of $q=1$ resonant surface is shown $\left(\rho_{\text {pol }}=0.25-0.3\right)$.

Spatial structure of the modes could be determined by mid-plain SXR camera I at the lowfield side of the tokamak. The primary mode at the frequency $f_{1}$ gives classical signature of the $(1,1)$ mode with one minimum in the plasma center as shown in figure 4 . Second harmonic of this mode is one order of magnitude smaller and shows $m=2$ (two minima) structure at the double frequency $\left(2 \cdot f_{1}\right)$. The most interesting question is the location and the spatial structure of the second mode $\left(f_{2}\right)$. This mode is always inside the $\mathrm{q}=1$ resonant surface as shown in Fig.4 and has a comparable amplitude to $(2,2)$ harmonic. The mode signal shows no local minima which points out $m=0$ structure. Such an observation is a preliminary result for the mode structure and a more detailed combined analysis is required, because the amplitude of the mode is small and toroidal periodicity cannot be determined from the SXR and ECE diagnostics for these shots. The precise nature of this mode in terms of an MHD perturbation is at present not clear, but will be addressed in a further work. An additional SXR camera at different toroidal position is installed inside ASDEX-U and will be used to estimate the toroidal mode numbers in the future experiments.

It should be mention here that depending on the mode amplitude, safety factor profile and the coupling of various resonances two different crash scenarios are possible:

- The strong coupling and/or large amplitudes of the perturbations can stochastize the entire core region of the plasma and completely destroy the flux surfaces.

- A weak coupling and/or small amplitudes can stochastize only the region around the separatrix of the $(1,1)$ mode which would also be sufficient for the sawtooth crash. In this case, confined plasma core is pushed out of the plasma center through the local (poloidal and toroidal) stochastic zone.

The island itself is always non-stochastic, as was shown in Ref.[7].

\section{Conclusions}


On the basis of analysis of the SXR and ECE data we have demonstrated that the sawtooth crash in magnetically confined plasmas in the ASDEX Upgrade tokamak shows clear signatures of the transition via quasiperiodicity to chaos. The chaotic stage itself is too short to be resolved, but all the signatures of the quasiperiodic transition phase are present:

1. The frequency spectrum (both in SXR and ECE) has two frequencies. Ratio between these frequencies is equal to the conjugate golden ratio (within error bars of the measurements). Other peaks are linear combinations of the two primary frequencies.

2. In contrast to many other systems, these two frequencies appear naturally in the sawtooth crash which means that the system approaches chaos in the most energetically favorable way (the most irrational frequency ratio).

3. $2 \mathrm{D}$ and $3 \mathrm{D}$ reconstructions of the system trajectory using delay coordinates show the transition from a purely periodic to strongly quasiperiodic behavior.

4. Strong increase of the low frequency part of the spectrum is observed just before the crash. This is also a typical feature of other quasiperiodic transition experiments [10].

5. Sawteeth events from several different H-mode discharges were analyzed and give similar results. In particular, the frequency ratio is the same.

Our understanding of the sawtooth crash is the following: growth of the $(1,1)$ mode leads to the nonlinear phase of the evolution and the harmonics of the primary mode emerge in the system. At the same time, a bifurcation in the system excites the second mode with irrational frequency ratio with respect to the primary $(1,1)$ mode. The low frequency spectrum becomes filled with linear combination of these frequencies. The interaction between the modes increases which causes frequency-locking [16]. Such a locking induces reconnection and immediately after the start of the reconnection stochastisation develops. Stochastization of magnetic field lines appears only for a very short time period which is the crash phase itself. The island is not destroyed and the mode remains at the same position after the crash, but all the temperature is lost during the stochastic phase.

We think that a sawtooth crash has a universal stochastic character and is closely related to the enhanced transport by the magnetic stochasticity. We have shown that in ASDEX-U not only the perturbations are sufficiently large for stochastization (as shown in Ref.[7]) but also signal dynamic before the sawtooth crash has all signatures of the transition to stochastic stage. We expect that in the sawteeth with long $(1,1)$ precursor phase (and thus long quasiperiodic phase) similar behaviour should be found also in other tokamaks.

\section{Acknowledgments}

The authors are indebted to Prof. K. Lackner for helpful discussions about the sawtooth phenomenon.

\section{References}

[1] S. Goeler, S. Stodiek, W. Sauthoff, Phys. Rev. Let. 33, 1201 (1974).

[2] B. B. Kadomtsev,Soviet Journal of Plasma Physics, 1, 710 (1975).

[3] F.M. Levinton, L. Zakharov, S.H. Batha, J. Manickam, M.C. Zarnstorff, Phys.Rev.Lett.,72,2895 (1994).

[4] M.Yamada, F.M. Levinton, N. Pomphrey, R.Budny, J.Manickam, Y. Nagayama, Physics of Plasmas, 1, 3269 (1994)

[5] H.Soltwisch, Rev. Sci. Inst., 59, 1599 (1988).

[6] A. Letsch, H. Zohm, F. Ryter, W. Suttrop, A. Gude, F. Porcelli, C. Angioni and I. Furno, Nucl. Fusion 42, 1055 (2002). 
[7] V. Igochine, O. Dumbrajs, H. Zohm, A. Flaws Nucl. Fusion 47, 23-32A (2007).

[8] A. J. Lichtenberg, Nucl. Fusion 24, 1277 (1984).

[9] A. J. Lichtenberg, K. Itoh, S.-I. Itoh, A. Fukuyama , Nucl. Fusion 32, 495 (1992).

[10] H. G. Schuster and W. Just, "Deterministic Chaos", $4^{\text {th }}$ edition, WILEY Verlag, Germany, 2005

[11] S. Martin et. al. Phys. Rev. Let. 53, 303 (1984).

[12] D. Rand et. al. Phys. Rev. Let. 49, 132 (1982).

[13] J. Argyris, G. Faust and M. Haase, "An exploration of Chaos”, North-Holland, 1994

[14] A. P. Fein et. al., Physica Scripta , T9, 79 (1985).

[15] A. Brandstater et. al. Phys. Rev. Let. 51, 1442 (1983).

[16] R. Hilborn, "Chaos and Nonlinear Dynamics: An Introduction for Scientists and Engineers", Oxford University Press, 2000

[17] H.K. Park, N.C. Luhmann, Jr., A.J.H. Donne, I.G.J. Classen, C.W. Domier, E. Mazzucato, T. Munsat, M.J. van de Pol , Z. Xia, and TEXTOR team, Phys. Rev. Let. 96, 195003 (2006).

[18] H. K. Park, A. J. H. Donne, N. C. Luhmann, Jr., I. G. J. Classen, C.W. Domier, E. Mazzucato, T. Munsat, M. J. van de Pol,Z. Xia, and TEXTOR Team, Phys. Rev. Let. 96, 195004 (2006).

[19] J. A. Wesson, Plasma Physics and Control Fusion 28(1A), 243 (1986).

[20] Y. Nishimura, J. D. Callen, and C. C. Hegna, Physics of Plasmas, 6, 4685 (1999).

[21] O. Dumbrajs, V. Igochine, H. Zohm, Nucl. Fusion 48 (2008) 024011 (7pp)

[22] E. Lazzaro et. al. , Physics of Plasmas, 9, 3906 (2002).

[23] S. Martin et. al. Phys. Rev. Let. 56, 1522 (1986). 